\title{
Sclerosing Meningioma : Radiological and Clinical Characteristics of 21 Cases
}

Ho Kang, M.D., ${ }^{1}$ Jin Wook Kim, M.D., Ph.D., ${ }^{1}$ Young-Bem Se, M.D., ${ }^{1}$ Yun-Sik Dho, M.D., ${ }^{1}$ Seung Hong Choi, M.D., ${ }^{2}$ Sung-Hye Park, M.D. ${ }^{3}$

Departments of Neurosurgery, ${ }^{1}$ Radiology, ${ }^{2}$ Pathology, ${ }^{3}$ Seoul National University Hospital, Seoul, Korea

\begin{abstract}
Objective : A rare subtype of meningioma, sclerosing meningioma is not included in the current World Health Organization classification of meningiomas and is classified into the category of other morphological variation subtypes. Sclerosing meningioma is often misdiagnosed to other non-benign meningioma or malignant neoplasm, so it is important to diagnose sclerosing type correctly. We analyzed the radiological and clinical characteristics of a series of sclerosing meningiomas.

Methods : Twenty-one patients who underwent surgery in one institute with a histopathologically proven sclerosing meningioma were included from 2006 to 2014. Eighteen tumors were diagnosed as a pure sclerosing-type meningioma, and 3 as mixed type. Magnetic resonance image was taken for all patients including contrast enhancement image. Computed tomography (CT) scan was taken for 16 patients. One neuroradiologist and 1 neurosurgeon reviewed all images retrospectively.

Results : In the all 16 patients with preoperative CT images, higher attenuation was observed in the meningioma than in the brain parenchyma, and calcification was observed in 11 (69\%). In 15 of the 21 patients (71\%), a distinctive very low signal intensity appeared as a dark color in T2-weighted images. Nine of these 15 tumors (60\%) exhibited heterogeneous enhancement, and 6 (40\%) exhibited homogeneous enhancement that was unlike the homogeneous enhancing pattern shown by conventional meningiomas. Ten patients had a clear tumor margin without peritumoral edema.

Conclusion : Although these peculiar radiological characteristics are not unique to sclerosing meningioma, we believe that they are distinctive features that may be helpful for distinguishing sclerosing meningioma from other subtypes.
\end{abstract}

Key Words : Meningioma · Magnetic resonance imaging.

\section{INTRODUCTION}

Sclerosing meningioma was first described in 1989 as a distinctive histological subtype of meningioma ${ }^{1}$. The histopathological features of sclerosing meningioma indicate that it is a meningioma with abundant collagenous tissues within extensive hyalinized areas and scattered small foci of classic meningioma histology ${ }^{4}$. Sclerosing meningioma is often misdiagnosed as a nonbenign meningioma or other malignant neoplasm because it tends to exhibit more severe peritumoral edema and greater invasion into the brain parenchyma compared with other conventional meningiomas ${ }^{1,6)}$. However, because of its rarity and lack of clinical information, sclerosing meningioma is not included in the current World Health Organization (WHO) classification and is categorized into one of the other morphological variation subtypes ${ }^{4)}$.

Only a few reports have discussed the clinical and histopath- ological characteristics of this rare tumor ${ }^{1,6,7,16,17)}$. Because of the paucity of reported cases, the radiological features of sclerosing meningioma are not well documented. It is a little known that calcification is more frequently observed on computed tomography (CT) images comparing to other meningioma subtypes ${ }^{6}$.

Therefore, the aim of this study was to investigate the clinical and radiological characteristics of sclerosing meningioma by performing a systematic analysis of cases of this rare tumor subtype.

\section{MATERIALS AND METHODS}

We retrospectively evaluated patients who had been surgically treated for a meningioma from 2006 to 2014. A total of 851 cases were histologically confirmed as a meningioma, and 21 of these with the sclerosing subtype were included in this study. The patients' clinical, radiological, histopathological, and surgical records were obtained from our electronic database and were

- Received : June 22, 2016 • Revised : August 12, 2016 •Accepted : August 30, 2016

- Address for reprints : Jin Wook Kim, M.D., Ph.D.

Department of Neurosurgery, Seoul National University Hospital, 101 Daehak-ro, Jongno-gu, Seoul 03080, Korea

Tel : +82-2-2072-2862, Fax : +82-2-744-8459, E-mail : wook616@hanmail.net

- This is an Open Access article distributed under the terms of the Creative Commons Attribution Non-Commercial License (http://creativecommons.org/licenses/by-nc/3.0) which permits unrestricted non-commercial use, distribution, and reproduction in any medium, provided the original work is properly cited. 
reviewed in detail. The median age of the patients (10 men, 11 women) was 55 years (range, 23-75 years).

Preoperative 1.5- or 3.0-T magnetic resonance (MR) imaging was performed, and T1-weighted, T2-weighted, and contrastenhanced T1-weighted images were obtained for all patients. Preoperative CT scans were obtained from 16 patients. All imaging studies were reviewed retrospectively by 2 observers ( 1 neuroradiologist and 1 neurosurgeon). They evaluated the following radiological features : attenuation (high, isodense, low), calcification, bony destruction, and hyperostosis (absent versus present) in CT images, and T1- and T2-weighted signal intensity (high, isodense, low), enhancement pattern (homogeneous versus heterogeneous), and peritumoral edema (absent versus present) in MR images.

Histopathological investigations were performed by a neuropathologist.

\section{RESULTS}

\section{Illustrative case}

A 75-year-old man visited the outpatient clinic with a chief complaint of gradually deteriorating dizziness. Preoperative MR images revealed a $4-\mathrm{cm}$ round mass in the cerebellum, which had very low signal intensity in T2-weighted images and appeared as a heterogeneously enhancing lesion after contrast administration (Fig. 1A, B). Despite the large tumor size, definite peritumoral edema was absent. A preoperative CT scan showed a high-attenuation lesion with diffuse and scattered calcification (Fig. 1C). The primary preoperative radiological diagnosis was an extra-axial tumor such as a meningioma. However, an extremely rare tumor such as a melanocytoma could not be excluded because of the peculiar hypointensity in T2-weighted images.

In the intraoperative findings, the tumor had an entirely avascular nature and clear margin from the brain parenchyma. However, the tumor was very tough and hard, and could not be removed with surgical scissors or an ultrasonic aspirator at full power. Finally, the tumor was removed totally by using an electromagnetic field system (Fig. 1D).

The final histopathological diagnosis was WHO grade I sclerosing-type meningioma. Detailed microscopic analysis showed an absence of components such as increased cellularity, small cells with a high nuclear : cytoplasm ratio, prominent nucleoli, uninterrupted patternless growth, and necrosis. Mitosis and brain invasion were not identified, and the Ki-67 labeling index was $0.2 \%$.

\section{Summary of all cases}

The details of the clinical, histopathological, and radiological data of all consecutive 21 patients are summarized in Table 1. Histopathological examination of the 21 tumors showed that 18 were diagnosed as a pure sclerosing-type meningioma, and 3 were diagnosed as mixed type meningioma with meningothelial, transitional, and psammomatous types each.

Sixteen of the 21 patients had received preoperative CT scans, and all of these scans showed higher attenuation in the tumor than in the normal parenchyma. Calcification was observed in CT scans of 11 (69\%) of the 16 patients. Bony destruction and hyperostosis of the adjacent bone were observed in 3 and 2 patients, respectively.

These sclerosing meningiomas exhibited distinguishing radiological characteristics in MR images, especially T2-weighted images (Fig. 2). In 15 of the 21 patients (71\%), distinctive very low signal intensity appeared as a dark color in T2-weighted images. In 6 patients, only isodense signal intensity was found in T2weighted images. In 5 of these 6 patients, the tumor exhibited a homogeneous enhancement pattern resembling a conventional meningioma, whereas the 15 tumors with dark signal intensity in T2-weighted images exhibited heterogeneous enhancement in 9 patients (60\%) and homogeneous enhancement in $6(40 \%)$. In most of the tumors with heterogeneous enhancement, the pattern of enhancement was similar to that of the illustrative case; this pattern resulted from the stronger peripheral enhancement in contrast to the weak enhancement in the inside of the tumor. In 10 patients (48\%), there was a clear tumor margin without peritumoral edema. Among the other 11 patients with perilesional edema, 7 tumors exhibited small amount of peritumoral edema and 4 exhibited moderate to severe peritumoral edema.

Eight of 21 patients underwent subtotal resection (STR), 12 patients did gross total resection (GTR), and another patient did near total resection. Six of 8 STR patients got adjuvant gamma
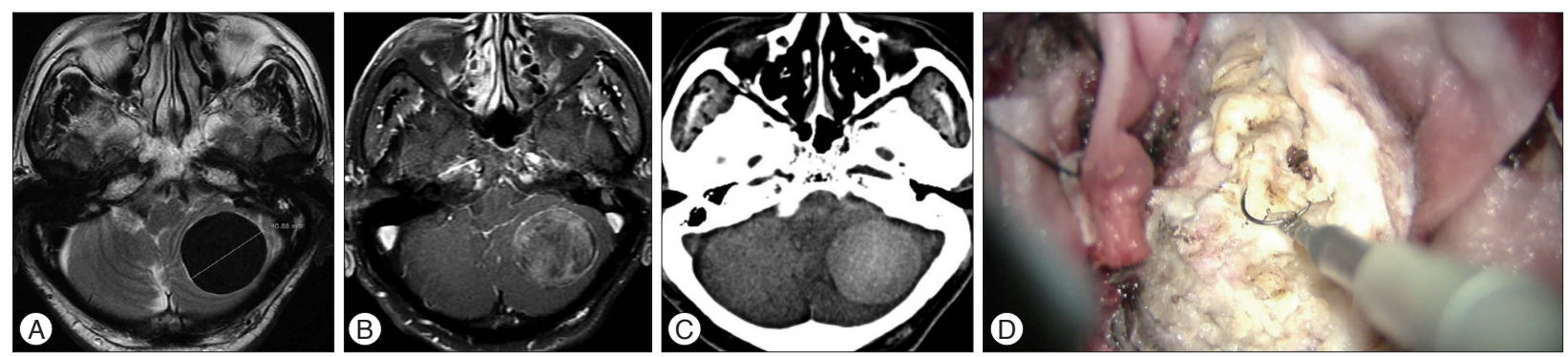

Fig. 1. Illustrative case. A and B : Preoperative MR images revealed $4 \mathrm{~cm}$ size round mass in cerebellum which was very low signal intensity on T2weighted image without peritumoral edema and heterogeneously enhancing lesion after contrast administration. C : Preoperative CT scan showed high attenuated lesion with diffuse and scattered calcification. D : Intraoperative photograph shows entirely avascular nature with yellowish color and clear margin to brain parenchyme. Because the tumor was very tough and hard, the tumor was removed totally by using an electromagnetic field system. 


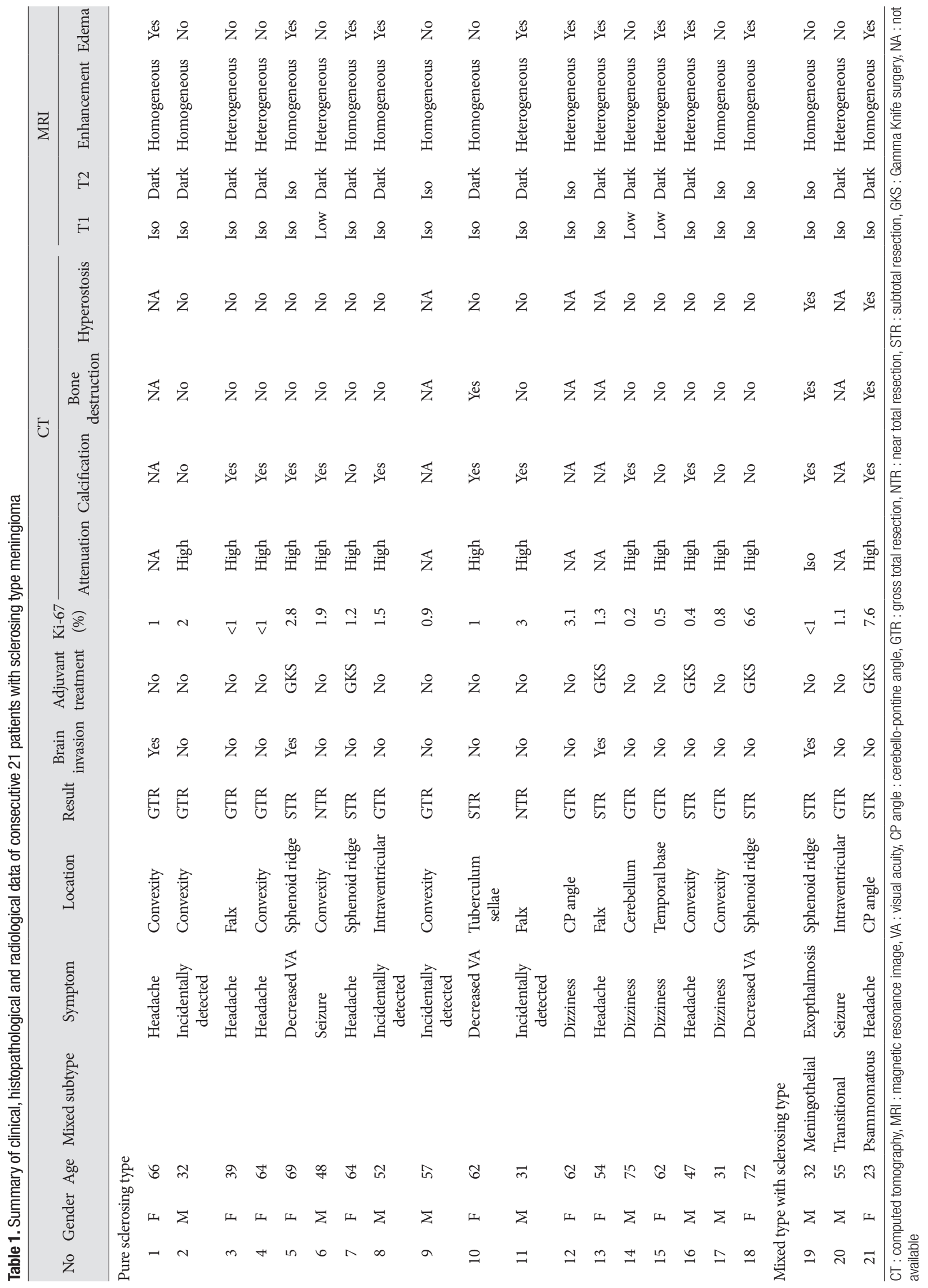




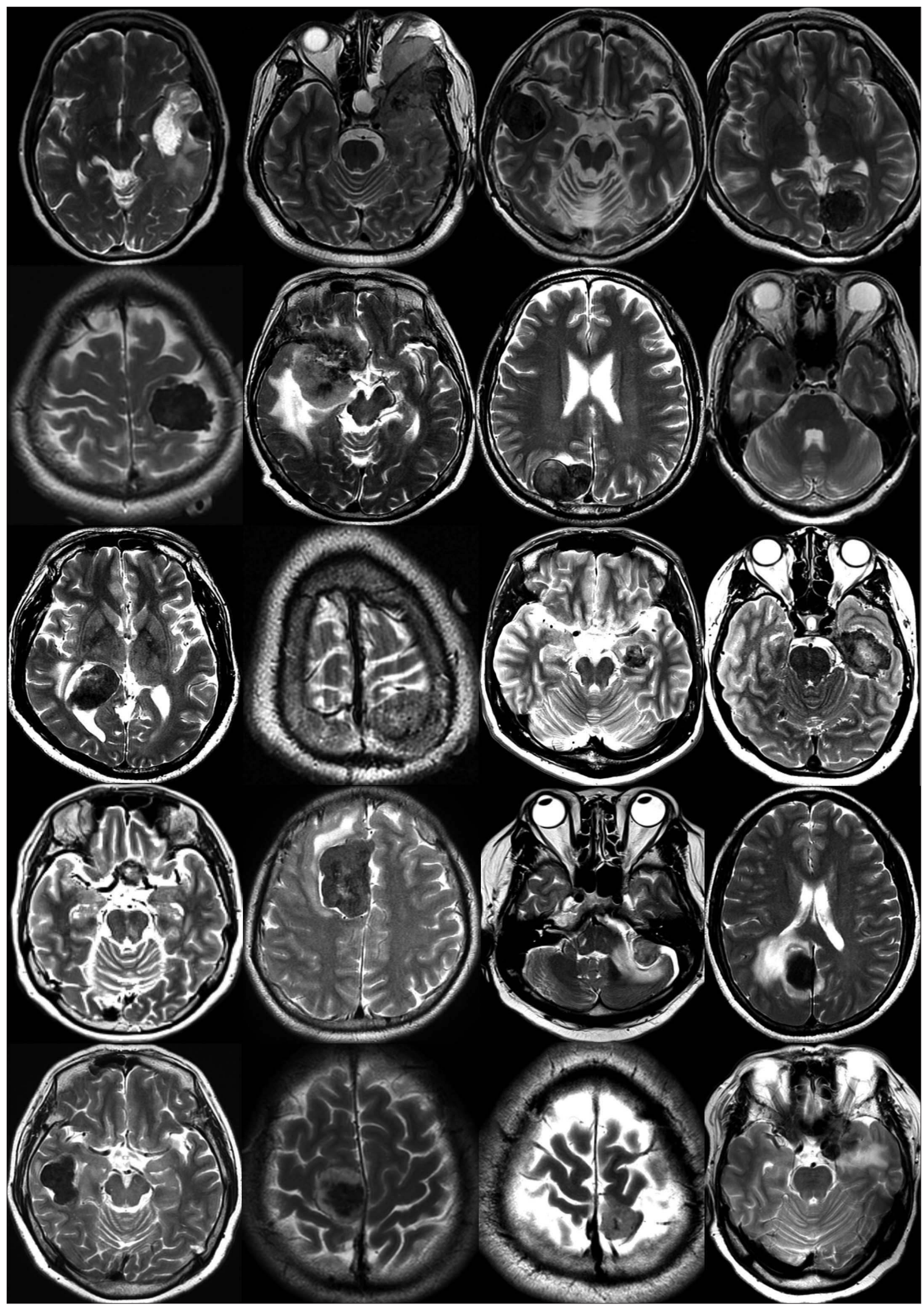

Fig. 2. T2-wheighted MR images of all patients with sclerosing meningioma except for the illustrative case. Among 21 patients, 15 patients (71\%) have their distinctive very low signal intensity which seemed to be nearly dark color on T2-weighted images whereas isodense signal intensity on T2 images was found in only 6 patients. Furthermore, the majority of sclerosing meningioma revealed absent or minimal extent of peritumoral edema. Moderate to severe peritumoral edema found in only 4 patients. 
knife surgery (GKS) for residual tumor.

All 21 tumors were diagnosed as WHO grade I benign meningioma. Brain invasion was found in only 4 patients (19\%), and the Ki-67 labeling index was $\leq 2 \%$ for all but 4 patients.

\section{DISCUSSION}

Many studies have reported relationships between radiological characteristics of primary brain tumors such as oligodendroglioma, astrocytoma, neuroma and meningioma and their subtypes and, by extension, their molecular subtypes ${ }^{2,10,14,19,20)}$. Similarly, a few studies have revealed a close relationship between the radiological features and the subtype of meningio$\mathrm{ma}^{3,8,9,15)}$. Kaplan et al. ${ }^{8}$ investigated the distinguishing imaging findings of 4 subtypes of meningioma (angioblastic, fibroblastic, meningothelial, and transitional) and reported differences in the signal intensity of the tumor according to subtypes. They introduced correct histological subgrouping of these tumors based on the MR signal intensity characteristics was possible in $80 \%$. Maiuri et al. ${ }^{13)}$ investigated correlation of pathologic feature of several types of meningioma and MR findings. They concluded that T2-weighted image are more useful to predict the characteristics of meningioma and meningioma hyperintense on T2 are usually soft and more vascular while hypointense tumor on T2 have more hard consistency and large amount of collagen stroma. Also, Several suptypes of meninigioma have been revealed about their characteristic radiological feature as microcystic meningioma ${ }^{15}$. Most of the cases in the series by Paek et al. ${ }^{15)}$ showed distinguishing radiological feature of microcystic meningioma markedly high signal intensity with severe peritumoral edema in T2-weighted images.

There are few reports about sclerosing meningioma because of its rarity. As noted above, the tumor has not been categorized in the current WHO classification of meningioma, and is instead categorized as one of the other morphological variation subtypes. Most reports on sclerosing meningioma have focused on the pathological findings. Kim et al. ${ }^{11)}$ reported that the pathological features of sclerosing meningioma include cellular elongation and a paucicellular collagenous whorl-like pattern. Im et al. ${ }^{7}$ reported that the pathological characteristics of sclerosing meningioma include extensive collagen deposition and an intermingled small population of spindle-shaped or round cells with clear cytoplasmic halos, which give a 'fried egg' appearance, with a typical meningothelial whorl pattern. They also reported that at least $70 \%$ of the total tumor volume should comprise collagen bundles for a meningioma to be diagnosed as the sclerosing variant ${ }^{7}$.

A noticeable finding of this study was the peculiar radiological characteristics in MR images, especially T2-weighted images. To our knowledge, no previous reports have summarized the radiological characteristics of sclerosing meningioma. Even if the very low signal intensity in T2-weighted images is not a pathognomonic feature of sclerosing meningioma, $71 \%$ of the cases (15 of 21 patients) in our series had a markedly low intensity, and rest of them showed nearly a dark signal in T2-weighted images (Fig. 2). Elster et al. ${ }^{3)}$ reported that the fibroblastic or transitional element of meningioma appears hypointense in T2-weighted images. This low signal intensity of meningioma in T2-weighted images is considered to reflect the psammomatous calcification and abundant acellular collagen-rich portion. Sclerosing meningioma is known to have a markedly higher proportion of collagen bundles and calcification than other meningioma subtypes. Thus, the sclerosing subtype may be characterized by marked hypointensity in T2-weighted images.

A few reports have shown that a characteristic feature of sclerosing meningioma is brain invasion ${ }^{5,7,16)}$. However, in our series, only 4 (19\%) of 21 cases involved brain invasion. The prevalence of peritumoral edema, which is often misdiagnosed as a non-benign meningioma or other malignant neoplasm, was not much higher (52\%) in our series, but rather small extent in more than half of sclerosing meningioma with peritumoral edema ${ }^{1,6)}$.

About half ( 10 of 21 cases) of the sclerosing meningiomas in our study showed a heterogeneous enhancement pattern that resulted from the strong peripheral enhancement in contrast to the weak enhancement of the inside of the tumor. However, classic meningiomas usually exhibit a homogeneously enhanced pattern. Considering that this heterogeneous enhancement was detected more frequently in the cases of sclerosing meningioma with a dark signal in T2-weighted images, the heterogeneous enhancement pattern seems to be closely related to the histological characteristics of the psammomatous calcification and abundant acellular collagen-rich portion in sclerosing meningioma.

Attenuation exhibited by meningiomas observed in CT scans varies in tumor characteristics such as consistency, cellularity, and invasiveness. Stevens et al. ${ }^{18)}$ reported that the hard consistency of a meningioma is related to the high attenuation observed in CT scans. The very dense collagenous meningiomalike sclerosing type tends to exhibit high attenuation in CT scans $^{1,6,16)}$. All 16 patients in our series with a preoperative CT scan exhibited high attenuation in their tumors.

Only 12 of 21 patients underwent GTR, while 8 patients underwent STR. Tumors of STR cases located relatively more on skull base than GTR cases, 4 at sphenoid ridge, 1 at tuberculum sellae, and 1 at cerebellopontine angle. The rest of the 2 patients had tumors convexity and falx, respectively. As described above, low signal of sclerosing meningioma on T2-weighted image reflects psammomatous calcification and abundant acellular collagen-rich ${ }^{7}$. The histopathological character could make sclerosing meningioma firm and hypovascular as our illustrative case. Therefore especially during resection of meningioma which has these characteristics on skull base, surgeon cannot resect tumor resolutely nearby important neurovascular structures. However, GTR might be not obligatory if intraoperative neurovascular injury was strongly possible. Because sclerosing meningioma is histopathologically benign, WHO grade 1 . Although outcome after adjuvant gammaknife radiosurgery specifically for scleros- 
ing meningioma has never been reported, adjuvant GKS for residual tumor could be considered for sclerosing meningioma same as other meningiomas ${ }^{12)}$.

\section{CONCLUSION}

Although these peculiar radiological characteristics are not unique to sclerosing meningiomas, we propose that characteristics of markedly hypointensity on T2-weighted image and heterogenous enhancement may be helpful for distinguishing this type of meningioma from other subtypes. These findings from the detailed analysis of 21 cases may provide insights into the nature of this rare tumor.

\section{References}

1. Davidson GS, Hope JK : Meningeal tumors of childhood. Cancer 63 : 1205-1210, 1989

2. Earnest F 4th, Kelly PJ, Scheithauer BW, Kall BA, Cascino TL, Ehman RL, et al. : Cerebral astrocytomas : histopathologic correlation of MR and CT contrast enhancement with stereotactic biopsy. Radiology 166 : 823-827, 1988

3. Elster AD, Challa VR, Gilbert TH, Richardson DN, Contento JC : Meningiomas : MR and histopathologic features. Radiology 170(3 Pt 1) : 857-862, 1989

4. Fukushima S, Narita Y, Yonezawa M, Ohno M, Arita H, Miyakita Y, et al. : Short communication : sclerosing meningioma in the deep sylvian fissure. Brain Tumor Pathol 31 : 289-292, 2014

5. Haberler C, Jarius C, Lang S, Rössler K, Gruber A, Hainfellner JA, et al. : Fibrous meningeal tumours with extensive non-calcifying collagenous whorls and glial fibrillary acidic protein expression : the whorling-sclerosing variant of meningioma. Neuropathol Appl Neurobiol 28 : 42-47, 2002

6. Hope JK, Armstrong DA, Babyn PS, Humphreys RR, Harwood-Nash DC, Chuang SH, et al. : Primary meningeal tumors in children : correlation of clinical and CT findings with histologic type and prognosis. AJNR Am J Neuroradiol 13 : 1353-1364, 1992

7. Im SH, Chung CK, Cho BK, Kim MK, Chi JG : Sclerosing meningioma : clinicopathological study of four cases. J Neurooncol 68 : 169-175, 2004
8. Kaplan RD, Coons S, Drayer BP, Bird CR, Johnson PC : MR characteristics of meningioma subtypes at 1.5 tesla. J Comput Assist Tomogr 16 : 366-371, 1992

9. Kim BW, Kim MS, Kim SW, Chang CH, Kim OL : Peritumoral brain edema in meningiomas : correlation of radiologic and pathologic features. J Korean Neurosurg Soc 49 : 26-30, 2011

10. Kim JW, Park CK, Park SH, Kim YH, Han JH, Kim CY, et al. : Relationship between radiological characteristics and combined $1 p$ and $19 q$ deletion in World Health Organization grade III oligodendroglial tumours. J Neurol Neurosurg Psychiatry 82 : 224-227, 2011

11. Kim NR, Im SH, Chung CK, Suh YL, Choe G, Chi JG : Sclerosing meningioma : immunohistochemical analysis of five cases. Neuropathol Appl Neurobiol 30 : 126-135, 2004

12. Kollová A, Liscák R, Novotný J Jr, Vladyka V, Simonová G, Janousková L : Gamma Knife surgery for benign meningioma. J Neurosurg 107 : 325-336, 2007

13. Maiuri F, Iaconetta G, de Divitiis O, Cirillo S, Di Salle F, De Caro ML : Intracranial meningiomas : correlations between MR imaging and histology. Eur J Radiol 31 : 69-75, 1999

14. Megyesi JF, Kachur E, Lee DH, Zlatescu MC, Betensky RA, Forsyth PA, et al. : Imaging correlates of molecular signatures in oligodendrogliomas. Clin Cancer Res 10 : 4303-4306, 2004

15. Paek SH, Kim SH, Chang KH, Park CK, Kim JE, Kim DG, et al. : Microcystic meningiomas : radiological characteristics of 16 cases. Acta Neurochir (Wien) 147 : 965-72; discussion 972, 2005

16. Pope LZ, Tatsui CE, Moro MS, Neto AC, Bleggi-Torres LF : Meningioma with extensive noncalcifying collagenous whorls and glial fibrillary acidic protein expression : new variant of meningioma diagnosed by smear preparation. Diagn Cytopathol 28 : 274-277, 2003

17. Sheikh BY, Siqueira E, Dayel F : Meningioma in children : a report of nine cases and a review of the literature. Surg Neurol $45: 328-335,1996$

18. Stevens JM, Ruiz JS, Kendall BE : Observation on peritumoural oedema in meningioma. Part I : distribution, spread and resolution of vasogenic oedema seen on computed tomography. Neuroradiology 25 : 71-80, 1983

19. Walker DG, Kaye AH : Diagnosis and management of astrocytomas, oligodendrogliomas and mixed gliomas : a review. Australas Radiol 45 : 472-482, 2001

20. Zlatescu MC, TehraniYazdi A, Sasaki H, Megyesi JF, Betensky RA, Louis $\mathrm{DN}$, et al. : Tumor location and growth pattern correlate with genetic signature in oligodendroglial neoplasms. Cancer Res 61 : 6713-6715, 2001 